\title{
Stefan Bajohr Abbau der Staatsverschuldung - Eine Bedingung nachhaltiger Reformpolitik
}

Mit Überlegungen, wie die Überschuldung öffentlicher Haushalte aufgehalten werden kann, sind die Regierungen aller modernen Staaten beschäftigt. Damit steht die keynesianische Verbeißung auf dem Prüfstand, durch gezielte Kreditfinanzierung seien wirtschaftliche Probleme in den Griff zu bekommen. Der Autor entwirft ein Konzept zur Haushaltssanierung, in dem das Recht zur Kreditaufnabme am Kapitalmarkt auf Fälle konjunkturbedingter Mindereinnahmen und unvorbergesebener Sondersituationen beschränt ist.

Die Red.

\section{Vorneweg}

16 Jahre Koalition aus Kabinett und Kapital hinterlassen nicht nur bei Kindern und Jugendlichen, bei den sozial Schwachen, den ArbeitnehmerInnen und den Erwerbslosen Spuren von Verwirrung und Verwüstung. Auch in der Wirtschafts- und Finanzpolitik haben Konservarive und Liberale ein Desaster angerichtet. Am eindrücklichsten zeigt sich die Finanzkrise an den 2,2 Billionen DM, mit denen der öffentliche Gesamthaushalt verschuldet ist. Dafür mußten 1997 rd. 136 Mrd. DM an Zinszahlungen geleistet werden' - eine Summe, die für Arbeitsmarktprogramme oder den ökologischen Umbau sinnvoller verwender werden könnte. Daß sie den öffentlichen Haushalten fehlt, belegen die hektischen und zum Teil sozialpolitisch einschneidenden Kürzungsaktivitäten im Bund, in den Ländern und Gemeinden. Eine auf Reformen abzielende Politik wird an diesem gefährlichen Zustand rasch Änderungen herbeiführen müssen; ansonsten werden Reformprojekte, von denen nur die allerwenigsten kostenlos zu haben sind, stets an den finanziellen Widrigkeiten scheitern.

\section{Schulden wachsen dynamisch}

Seit nunmchr 26 Jahren wird ununterbrochen - nicht nur auf Bundesebene, sondern auch bei den nachgeordneten Gebietskörperschaften - ein beachtlicher Schuldenberg angehäuft. Ihre Verstärkung erfuhr die Staatsverschuldung in vicr Schüben, von denen die Finanzierung der Einheitsfolgen den stärksten Impuls auslöste (vgl. 'Tab. I). ${ }^{2}$

I Deutsche Bundesbank: Monatsbericht Juni $199^{\circ}, 54^{\circ}, 56^{\circ}$.

2 Hemmt oder stützt dic Staatsverschuldung die Wirtschafesentwicklung in Deutschland?, in: DIW-Wochenbericht 61. 1994, 34 I-349, hier 341. 
Dabei blieben Warnungen vor dem Marsch in die Überschuldung weitgchend unberücksichtigt - was um so leichter fiel, als »der Übergang von wenig zu viel und von viel zu zuviel (...) nicht mit Wegmarkierungen versehen « ist, sondern sich "stufenlos " vollzieht. ${ }^{3}$ Inzwischen hat die Gesamtverschuldung bedrohliche Ausmaße erreicht. ${ }^{4}$ Der Schuldenstand des öffentlichen Gesamthaushalts übertrifft dessen Volumen untrügliches Indiz dafür, daß der Weg in die Staatsüberschuldung längst beschritten ist. ${ }^{3}$

Die volle Wahrheit ist noch bitterer: $Z$ u den "offiziellen « Schulden müssen, erstens, Risiken aus Eventualverbindlichkeiten ${ }^{6}$ hinzugerechnet werden, die ohne Vorwarnung mit mehrstelligen Milliardenbeträgen zu Buche schlagen können. Zweitens: Sondervermögen sind aus dem Bundeshaushalt ausgegliedert; wenn die Legislative von der Er?nächtigung aus Art. is Abs. 2 GG Gebrauch gemacht hat, unterliegt ihre Kreditaufnahme nicht mehr den Obergrenzen des Verfassungsrechts. ${ }^{7}$ Ende 1997 waren die Sondervermögen mit rd. $516 \mathrm{Mrd}$. DM verschuldet; das entsprach rd. $23 \%$ des Schuldenstandes. ${ }^{8}$ Es existiert, drittens, auch kein Überblick über die eingegangenen Verpflichtungen öffentlicher oder solcher privatrechtlich verfaßter Unternehmen, für die die öffentlichen Haushalte letzten Endes geradestehen müssen. ${ }^{9}$ Darüber hinaus haben die sogenannten Verwaltungsschulden einen solchen Umfang angenommen, daß »ihre ökonomischen Wirkungen auf den Haushalt und die Volkswirtschaft keineswegs als Quantité négligeable eingeschätzt werden können «. ${ }^{10}$ Über die Risiken, die öffentlichen Haushalten aus Leasing- und Privatfinanzierungsverträgen (z. B. beim Verkehrswegebau) drohen, "liegt - fünftens - ebenfalls keine vollständige Übersicht vor. Sechstens finden auch die dynamisch wachsenden Versorgungsleistungen für BeamtInnen (RichterInnen, BerufssoldatInnen) bisher keinen Niederschlag in einer umfassenden Bilanz öffentlicher Verbindlichkeiten. ${ }^{12}$

Die jährliche Nettokreditaufnahme unterlag in der Vergangenheit erheblichen - auch konjunkturbedingten - Schwankungen (vgl. Tab. 2), weist insgesamt aber steigende Tendenz auf. Die dadurch bewirkte weitere Anhäufung des Schuldenberges verursacht Steigerungen des Schuldendienstes, die wiederum die Handlungsspielräume künftiger Politik einschränken. ${ }^{\text {'3 }}$

In bezug auf die Tragbarkeit der Staatsverschuldung aussagekräftiger als absolute Zahlen sind indes relative Rechengrößen. Zur Beantwortung der Frage, ob eine gegebene öffentliche Schuld "tragbar « - nicht zu verwechseln mit "wünschenswert « - ist, ${ }^{14}$ werden in der wissenschaftlichen und der politischen Debatte dic verschic-

3 Handelsblatt vom 10./1 1. 5. 1991 .

4 Vgl. Lüder 1994.

s Vgl. auch Mußgnug 1996, 74

6 Vgl. Sturm 1993, ir.

7 Hierbei werden in der Regel nicht einnal Infrastrukturprojekte finanzıert, sondern Ausgaben der laufenden Rechnung. Vgl. Schlesinger/Weber/Zicbarth 1993, 271.

8 Vgl. Deutsche Bundesbank: Monatsbericht Juni $1998,5.56^{*}, 57^{*}$

9 Vgl. Puhl 1996, s02.

10 W. Hofling 1993,31

is Vgl. Edinger $1997,251,253 \mathrm{f}$

12 Vgl. Bericht der Bundesregierung ùber dic im Kalenderjahr 1993 erbrachten Versorgungsleistungen im offentlichen Dienst sowie uber dic Entwuicklung der Versorgungsausgaben in den nachsten is Jahren, BTDrs. 13/5840. Danach wird sich die Zahl der VersorgungsempfangerInnen bis 2008 gegenuber 1994 um $39 \%$ auf 872.900 erhöhen (63). Die Versorgungsleistungen werden im gleichen Zeitraum um 72 bis $86 \%$ steigen ( 23 f.). Der Anteil der Versorgungsausgaben an den Steuereinnahmen wird im alten Bundesgebiet einschließlich West-Berlin von s auf bis zu 6,6\% zunehmen ( $3 r$ ).

${ }_{13}$ Das Bundesverfassungsgericht hat davor gewarnt, daß sich sein stetig wachsender Schuldensockel bildet, der schließlich die Fahigkeit des Staatshaushalts, auf die Probleme der Gegenwan und der Zukunfe zu reagieren, in Frage stelle $\alpha$ : BVerfGE 79, 311-357, hier 356; vgl, auch Karehnke 1973, 402; Brenner/Haury/ Lipp 1990, 245 .

14 Vgl. Singer 1996a, 34; Wiesc 1997, 57. 
densten Kombinationen angeboten. "Plausibel erscheinen in diesem Zusammenhang Überlegungen, denen zufolge eine öffentliche Schuld als tragbar zu bezeichnen ist, wwenn erwartet werden kann, daß irgendwann in der Zukunft Budgetüberschüsse erzielt werden, aus denen die Schuld getilgt werden kann «. Probleme ergeben sich demnach nnicht aus zeitwcilig hohen Defiziten, sondern (...) bei einer konstanten (...) Lücke zwischen öffentlichen Einnahmen und Ausgaben ".6 Wer allerdings auf den gesamtstaatlichen Primärsaldo blickt (vgl. Tab. 2), wird feststellen, daß eine Verwendung des durchaus vorhandenen Primärüberschusses zu Tilgungszwecken nicht stattfinden kann, weil er vom Zinsendienst vollständig aufgefressen wird. Mit anderen Worten: Die aufgetürmten Schulden haben zur Folge, daß aus Stcuern und Gebühren weniger notwendige, nützliche oder wünschbare Leistungen erbracht werden können, "als wenn der Staat von vornherein auf Kredite verzichtet hätte «. ${ }^{17}$ Mit welcher Konsequenz die Zinszahlungen wachsende Anteile am Steueraufkommen okkupieren, zeigt die Entwicklung der Zins-Steuer-Quote ${ }^{18}$ (vgl. Tab. 3). Übertrieben pessimistisch sind zweifellos Modellrechnungen, mit deren Hilfe ermittelt werden soll, in welchem Jahr das Steueraufkommen vollständig von den Zinsausgabeverpflichtungen beansprucht werden wird. ${ }^{\prime 9}$ Die Daten legen allerdings nahe, daß wir einen Punkt erreicht haben, an dem nicht mehr allein die Nettokreditaufnahme die Gesamtverschuldung determiniert, sondern an dem die Zinsausgaben ihnen zur Seite treten: "Damit nährt sich die Verschuldung aus sich selbst heraus. ${ }^{20}$

Diese Fahrt in die Sackgasse der Schuldenpolitik, an deren Ende sich eine tiefe Schlucht auftut ${ }^{21}$, hat sich trotz des verfassungsrechtlichen Verbots übermäßiger Kreditfinanzierung ${ }^{22}$ vollzogen. Die von der Verfassung gesetzten Grenzen ${ }^{23}$ vermochten es nicht, die Finanzkrise abzuwenden. Denn: Was den Bundeshaushalt angeht, so waren sämtliche Haushaltspläne in Einnahmen und Ausgaben ausgeglichen; die mit den jewreiligen Investitionsausgaben ${ }^{24}$ gesetzte Obergrenze für die Aufnahme von Krediten wurde bei der Verabschiedung des Haushalts in den 1 $980 e r$ und -9oer Jahren regelmäßig beachtet; die Nettokreditaufnahme lag (abgesehen von r 981 und 1997) unter den Investitionsausgaben; ob die Erfordernisse des "gesamtwirtschaftlichen Gleichgewichts" angemessen berücksichtigt waren, muß wegen der Unbestimmtheit dieses Verfassungsbegriffs ${ }^{25}$ und der prinzipiellen dogmatischen Offenheit der staatsschuldenrechtlichen Normen des Grundgesetzes ${ }^{26}$ an dieser Stelle unentschieden bleiben.

is Singer 1996a, 34, spricht von Tragbarkcih, "wenn die kunftigen Budgetuberschusse für eine Schuldentilgung ausreichen «; fur Wiese 1997, s6 ist ein Schuldenstand tragbar, wenn die Summe aus Steuer- und Defizitquote auf der Einnahmenseite der Staatsausgabenquote einschließlich der Zinsquote auf der Ausgabenscite entspricht. Kitterer 1996, 84 , will das Thema Tragbarkeit ausgedehnt wissen auf die Frage, ob die Folgen einer Konsolidierung (...) durch Steuern tragbar sind $\%$

$16 \mathrm{Vgl}$. Heinemann $1994, \mathrm{t} 2$.

17 Gantner 1979, 157 .

18 Hieran will Klaus Lider (mdl. Beitrag auf der Anhorung des Haushalts- und Finanzausschusses des Landtags Nordrhcin-Westfalen am 31. 0.1996 zur Staatsverschuldung, LT[NRW]-Drs. 12/383, 5) die z.umutbare Verschuldung messen. Gleichermaßen Hardt/Hiersemenzel/Keppler 1996, 26.

I9 $\mathrm{Vgl}$. Hofling 1993,264 .

20 Deutsche Bundcsbank: Monatsbericht Marz. 1997, 30. Vgl. auch Brenner/Haury/Lipp 1980, 245. Den Befund bestreitet Huffschmid 1997, 454

21 Zur Zeit bestehen fur die Länder des Euro keine konkreten Gefahren einer Wahrungszerruttung oder gar eines Staarsbankrotrs. Wahrungsschnitte sind jedoch in der Regel die logische Konsequenz ciner dauerhaften Schuldenpolitik (vgl. Becker 1998, 63).

22 Vgl. Piduch 1997, Art. irs Rdnr. 9, 26-31.

23 Vgl. Art. rog Abs. 2, Art. 110 Abs. i Satz 2 und Art. 11s Abs. 1 Satz 2 Halbs. 1 GG.

24 Zur Legaldefinition von Investitionen vgl. $\$ 10 \mathrm{Abs}$. $3 \mathrm{Nr} .2 \mathrm{HGrG}$; wortgleich in $\$ \mathrm{~L}_{3} \mathrm{Abs}$. $3 \mathrm{Nr} .2 \mathrm{BHO}$

25 Vgi. BVerfGE 79, 311, hier 338. Dic Unbestımmehcit bewirkt allerdings nicht dic Unbeachtlichkeit. Zu Reche weist Prokisch 1993, 137, darauf hin, daß es sich um einc Staatszielbestimmung handclt, die sals solche eine staatliches Handeln dirigierende Norm« darstellt.

$26 \mathrm{Vgl}$. Hofling 1993, 3. 
Diesbezügliche Zweifel sind allerdings insofern angebracht, als weder Bund noch Länder in Aufschwungphasen eine Konjunkrurausgleichsrücklage (\$s Abs. 2 StWG) gebildet haben, um - (zunächst) ohne Kreditaufnahme - auf eine Rezession reagieren zu können. Wie sehr sich Parlamente und Regierungen daran gewöhnt haben, ihre Haushalte durchweg mit Hilfe mehr oder minder großer Kredite zu finanzieren, erweisen die Finanzberichte. Darin weisen die Regierungen aller politischen Farben und Farbkombinationen Jahr für Jahr die geplante Nettoneuverschuldung für das jeweils kommende Jahrfünft aus - ohne daß irgend jemand die konjunkrurelle Entwicklung für so lange Zeiträume vorhersagen könnte. ${ }^{27}$ Das scheint inzwischen ohnehin recht gleichgültig zu sein: Wenn eine nStörung des gesamtwirtschaftlichen Gleichgewichts " sowohl in der Rezession als auch im Boom gegeben ist, ${ }^{28}$ dann kommen sogenannte Normallagen kaum mehr vor. Und dann berief sich die Bundesregierung in vergangenen Jahr - als es mit dem Haushaltsausgleich partout nicht klappen wollte - nicht einmal zu Unrecht auf die Ausnahmeregelung des Art. I is Abs. I Satz 2 Halbs. 2 GG, wonach selbst konsumtive Ausgaben kreditfinanziert werden dürfen, wenn dies »zur Abwehr « eben dieser Störung erforderlich scheint. ${ }^{29}$ Nun ist der Haushaltsplan, bei dem die Welt - formal betrachtet - noch in Ordnung scheint, das eine; das andere ist der Haushaltsvollzug. Hier ergibt sich bei Gegenüberstellung von Investitionsausgaben und Nettoneuverschuldung ein etwas anderes Bild. Es zeigt sich, daß die Kohl'schen Bundeshaushalte im Ist 1988, 1990, 1993 und 1996 die vom Grundgesetz auferlegte Kreditobergrenze verletzten. Erstaunlicherweise regte sich Widerstand hiergegen erst mit dem Haushaltsabschluß 1996: Die SPD-Bundestagsfraktion hat beim Bundesverfassungsgericht Klage eingereicht ${ }^{30}-$ wobei allerdings deutlich zutage tritt, daß es dabei nicht um ein Grundanliegen der Sozialdemokratie geht: Ansonsten würde der nordrhein-westfälische Finanzminister, beim Haushalts-Ist 1997 in der gleichen Lage wie sein Bonner Kollege im Jahr zuvor, nicht all'unisono mit diesem eine Geltung der verfassungsrechtlichen Kredithöchstgrenze für den Vollzug verneinen. Regierungen, gleich welcher Couleur, sind anscheinend kraft Amtes davon überzeugt, daß sie die von der Verfassung vorgegebenen Verschuldungsgrenzen einhalren - und wenn nicht, dann wird eben die Verfassung ins Sinnlose hinein interpretiert. ${ }^{3 t}$

Wenn trotzdem weithin Konsens besteht, daß die jährliche Nettoneuverschuldung zu hoch und der Umfang des aufgehäuften Schuldenberges besorgniserregend sei, weist dies auf die Unzulänglichkeit geltenden Verfassungsrechts hin. Mit gewisser Hoffnung haben deshalb manche die neue Grenzziehung angenommen, die der Vertrag von Maastricht brachte (Art. 104C EGV). ${ }^{32}$ Anders als im Grundgesetz gelten Ober-

27 Vgl. Gandenberger 1981,8 .

28 So z. B. Brenner/Haury/Lipp 1980, 242. Zu Recht bemangelt W. Scherf 1996, 377, den finanzverfassungsrechclichen Konstruktionsfehler, eine Kreditfinazzicrung nicht zumindest im Boom auszuschlicßen.

29 Das Bundesverfassungsgericht hat dic Berufung auf die Ausnahmeregelung insofern eingeengt, als es verlangt, daß die die Investitionsausgaben uberschießende Kreditaufnahme $\times$ nach Umfang und Verwendung gecignet sein $\propto$ müsse, dic Stórung abzuwehren (BVerfGE 79, 311, hicr: 339); wcgen der o.c. Unbestimmtheit bleibt die Letztentscheidung allerdings unüberprüfbar bei der jeweiligen Parlamentsmehrheit.

$30 \mathrm{Vgl}$. Das Parlament vom 7.11. 1997

3I Im Verein mit Waigel in Bonn und Schlcußer in Düsseldorf bestreiten eine Geltung von Art. is Abs. I Satz 2 GG auch für die Haushaltsausführung z. B. Patzig 1991, Art. 1 15, Rdnr. 25; Piduch 1997, Art. 115, Rdnr. 28; Maunz r997, Art. I1 5, Rdnr. 43. Fur die Geltung auch im Vollzug sprechen sich aus Friauf 1990, Rdnr. 43; Bundesrechnungshof 1994, 2 I f.; Tiemann 1995, 634; H. Fischer-Menshausen 1996, Rdnr. II; Müller 1996, 490; Blasius/Jahnz 1997, 32; Bröcker 1997, 79; Henneke 1997, 220; Heuer 1998, Art. r1 s, Rdnr. 10.

32 Die Defizitgrenzen getten nicht nur fur den Bund, sondern auch fur die Lander. Die Frage, zu welchen Anteilen sich Bund und Sozialversicherungen einerseits, Länder und Gemeinden andererseits verschulden dürfen und weiche Art dauerhafter Vereinbarung zwischen den Ebenen und unter den Länderrn eingegangen werden soll, wird derzeit ausgehandelt. Eine Gesetzgebungskompetenz besitzt der Bund in dieser Frage nicht. Vgl. Wehde 1997, 623; Schemmel 1997, 39 ff. 
grenzen nicht mehr nur für die Nettokreditaufnahme, sondern auch für den Schuldenberg selbst. ${ }^{33}$ Sie errechnen sich aus der Gegenüberstellung des jährlichen Haushaltsdefizits bzw. der Gesamtverschuldung mit dem Bruttoinlandsprodukt und drükken sich in Prozentwerten als Defizit- $(3 \%)$ oder Schuldenstandsquote $(60 \%)$ aus. ${ }^{34}$ Der »Pferdefuß dieser Übereinkunft besteht allerdings nicht allein darin, daß die nun geltenden Werte nicht Ergebnisse sorgfältiger Analyse sind, sondern ausgewürfelt scheinen (wenngleich neuerdings der Versuch unternommen wird, ihnen nachträglich wissenschaftliche Weihen zu verleihen) ${ }^{35}$; Zurückhaltung ist vor allem angebracht, weil sich in den Maastrichter Quoten eine Wachstumsorientierung manifestiert, die jedenfalls unter ökologischen Gesichtspunkten mit Skepsis betrachtet werden muß: ${ }^{6}$ Auch unter Bedingungen der Entkoppelung von Umweltzerstörung und Wirtschaftswachstum, wie manche sie neuerdings meinen feststellen zu können, birgt das Wachsen des Bruttoinlandsprodukts in sich selbst nachteilige Auswirkungen auf Klima und Umwelt. Fiskalisch enttäuschen die WWU-Kriterien insofern, als sie die weitere Anhäufung des Schuldenberges keineswegs verhindern; es muß bloß die entscheidende Bezugsgröße stärker zunehmen als die Neuverschuldung. Sogar wenn die Defizitquote sinken sollte, erwächst daraus keine Abzweigung aus der Sackgasse; auch in diesem Falle begibt sich die Gesellschaft wegen der zusätzlichen Zinslasten, die in der Tat »immer mehr zur Fron « werden, ${ }^{37}$ näher an den Abgrund.

Nur ein dauerhaftes Sinken der längerfristigen Kapitalmarktzinsen unter das Wirtschaftswachstum könnte Abhilfe schaffen. In der Realität verhält es sich seit Jahren allerdings genau umgekehrt. Wann die Kapitalmärkte hierauf mit Zinssatzverschlechterungen reagieren, ${ }^{38}$ die dann aller Voraussicht nach mit massiven Leistungskürzungen beantwortct würden, dürfte bloß noch eine Frage der Zeit sein.

\section{Senkung des Volkseinkommens und Umverteilung von unten nach oben}

Begonnen hat der Marsch in die Sackgasse der Überschuldung mit der keynesianischen Verheißung, die wirtschaftlichen Probleme in der Rezession/Depression mit gezielter Kreditfinanzierung in den Griff zu bekommen. Befürchtungen negativer Langfristeffekte wurden mit dem Versprechen auf cin "Schuldenparadox « abgetan, wonach aktives Deficit-spending in der Depression "zu Einnahmen- und Beschäftigungseffekten führt, die dem Staat Mehreinnahmen oder Ausgabenersparnisse verschaffen, die die ursprüngliche Staatsverschuldung kompensieren oder sogar überkompensieren ".39 Richtig angewendet, werde die antizyklische Konjunkturpolitik verschuldungsneutral ausfallen. Schulden- und Zinslastquote strebten festen, tragbaren Grenzwerten zu. ${ }^{\circ}$ Bereits in den x $960 e r$ Jahren war in Deutschland der Glaube an ein solches »ökonomisches Perpetuum mobile «" dermaßen übermächtig gewor-

33 Zusätzlich disziplinierend dürfe wirken, daß Art. 104c EGV den Ecofin-Rat ermächrigc, Maßnahmen gegeniber zu defizitfreudigen Mitgliedstaaten zu erlassen. Vgl. Hartmann I994, 18.

34 Auf unterschiedliche Ergebnisse je nach Verwendung von VGR oder Finanzstatistik kann hier niche cingegangen werden. Der Vertrag von Maastricht sicht die Anwendung der - großzügigeren - VGR vor. Vgl. auch Schmahl 1996, 93

3) Vgl. Kicterer $1996,82 \mathrm{~F}$.

36 Vgl. Stratmann-Mertens: mdl. Beitrag auf der Anhörung zur Staatsverschuldung, LT(NRW)-Drs. 1 $2 / 383$, 64.

37 Handelsblatt vom 22. 1. 1997.

38 Becker 1998 , 51 .

39 Oberhauser 1985,333 . Auf diese wundersame Vermehrung des Volkscinkommens sctzt z. B. auch Lerner $1964,112$.

40 Vgl. Domar 1944.

41 Ottnand 1996, 2. 
den, daß die keynesianische Wende sogar das Staatsschuldenrecht beeinflußte. Art. 109 GG wurde - regierungsoffiziell - mit dem Ziel umgeschrieben, »allen öffentlichen Haushalten eine verfassungsrechtliche Pflicht zur antizyklischen Haushaltsgestaltung “ aufzuerlegen..$^{42}$ Weil aber Eingriffe wie die Defizitfinanzierung »die Wiederkehr eines Zustandes kapitalistischer Prosperität behindern, die groß genug ist, um einen Haushaltsüberschuß zu ermöglichen «, ${ }^{43}$ versagt die keynesianische Praxis regelmäßig bei der Bildung der erforderlichen Rücklagen und dem Abbau der Verschuldung im Boom. ${ }^{44}$ Und so nimmt es nicht wunder, daß sich keine Regierung und kein Parlament an dic in Art. 109 Abs. 2 GG zum Ausdruck kommende Vorstellung einer konjunkturneutralen Verschuldungspolitik gehalten hat."s

Was als scheinbar sozial orientiertes Lösungsmodell innerhalb kapitalistischer Produktionsverhältnisse daherkam, erweist sich bei näherem Hinsehen als gigantische Umverteilung, die das Volksvermögen keineswegs mehrt, ${ }^{, 6}$ sondern ein immenscs Leistungsbilanzdefizit vorbereitet. ${ }^{47}$ Ursache dafür ist die wachsende Auslandsverschuldung, wobei vor allem die relativen Einflußgrößen Aufmerksamkeit verdienen (Tab. 4). 1980 entfielen rd. 9\% der Schulden auf GläubigerInnen im Ausland; 1997 waren es bereits rd. $32 \%$. Mit welcher Dynamik sich diese Entwicklung vollzieht, ist am Wachstum des Anteils von AuslandsgläubigerInnen beim Erwerb öffentlicher Schuldtitel aus Deutschland ablesbar: Entfielen auf sie 1980 nur rd. 40\% der Nettokreditaufnahme, so hat sich dieser Anteil bis zum vergangenen Jahr auf $82 \%$ gesteigert. Das macht deutlich, inwieweit sich bereits ein internationaler Staatsverschuldungsmarkt etabliert hat: Die Geldschöpfung wird tendenziell nicht mehr von Staaten vorgenommen, sondern von internationalen Finanzkomplexen ${ }^{48}$ in deren Abhängigkeit sich die nationalen Ökonomien begeben. ${ }^{49}$

Was die Auslandsverschuldung gegenwärtig an zusätzlicher Ressourcennutzung ermöglicht, wird später im Wege des Verzichts abbezahlt werden müssen. Der Staat befindet sich hier - anders als bei der Inlandsverschuldung - im gleichen Verhältnis zum/zur GläubigerIn wie der/die private Schuldnerln; ${ }^{\circ}$ und während der Schuldendienst die Inlandskonjunktur dämpft, verflüchtigt sich das überschüssige Geldkapital - auf der Suche nach der jeweils profitabelsten Anlageform - in der virtuellen Welt globalisierter Finanzmärkte.

Daß eine interpersonelle Einkommensumverteilung die logische Konsequenz der Inlandsverschuldung ist, wird von manchen vehement bestritten." Dabei ist gar nicht zu bezweifeln, daß es nicht SozialhilfeempfängerInnen, ArbeiternehmerInnen mit kleinen und mittleren Einkommen oder Kleingewerbetreibende sind, die dem Staat

42 BT-Drs. V/89o, 11. Obwohl das BVerfG meint, daß der "Entstehungsgeschichtc, den Vorstellungen und Motivationen $\propto$ im Vorfeld von Verfassungsanderungen nnicht unbedingt eine ausschlaggebende Bedeutung * zukomme (vgl. BVerfGE 45, 187-271, 227), liegt Nahamowitz 1990, 429, nicht ganz falsch, wenn cr den Findruck erweckt, als sei die keynesianische Interpretation von Art. 109 Abs. 2 GG die einzig zutreffende.

43 Mattick $1971,168$.

44 Vgl. Wacrin $1996,23 \mathrm{r}$

4 S Vil. Göke 1996, 2.

46 Vgl. Pohmer 1972, 159, 163.

47 Vgl. Richter/Wiegard r993, 372

$48 \mathrm{Vgl}$. Rugemer t995, ss.

49 Gleichsa:n als Palliativum weist z. B. die Bundesbank darauf hin, daß dahinter auch (ielder steckren, die via Steucrhinterzichung ihren Weg ins Ausland gefunden hätten und nun ins deutsche Finanzsysten zurückflössen (vgl. Deutsche Bundesbank: Monarsberiche März 1997, S. 28). Das ändert jedoch nichts daran, daß der Zinsendienst ans Ausland geleistet werden muß.

so Vgl. Gandenberger 1979a, 118 .

st Vgl. Andel 1969; Gandenberger 1979b, 139, 142; Gantner 1979, 154; Heun 1985, 27. Walturs (1967) meint sogar, daß die "A rmen " mehr Vortesle aus der Staatsverschuldung ziehen als die "Reichen". Vun der sozial

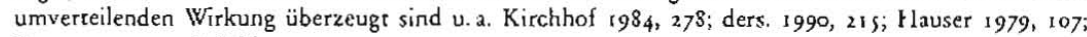
Rugemer 1995, 56; Schieren 1994, 207. 
Kredit gewähren, sondern in erster Linie Banken und Sparkassen, ${ }^{52}$ Versicherungen, einkommensstarke und vermögende Privatleutc sowie neuerdings auch Industriekonzerne." Und es ist offenkundig, daß diese Kredite Rückzahlungs- und Zinsverpflichtungen an eine Minderheit von GläubigerInnen auslösen, die von der Allgemeinhcit der SteuerzahlerInnen erfüllt werden müssen. Zur Rechtfertigung dieses umverteilenden Vorgangs wird angeführt, die KreditgeberInnen suchten sich ohne Staatsverschuldung Alternativen auf dem Kapitalmarkt und zögen dann daraus ihre Zinsgewinne. Insofern sei der Staatskredit nicht kausal für ihre Zinseinkommen. ${ }^{14}$ Dabei wird geflissentlich übersehen, daß die Alternative zur Kreditaufnahme (neben rigorosem Kaputtsparen) die Vermehrung der Steuereinnahmen ist - wobei keineswegs ausgemacht ist, daß Einnahmeverbesserungen aus Steuern in jeder politischen Situation zu Lasten der Masseneinkommen erfolgen müssen. Ob bei einer leistungsgerechten Anhebung der Steuern auf Einkommen und Vermögen die KreditgeberInnen überhaupt noch oder jedenfalls im heute gegebenen Umfang nach profitablen Anlagemöglichkeiten suchen müßten, wäre jedenfalls zu erörtern. Und weiter: $\mathrm{Zu}$ fragen ist nicht nach der Kausalität der Zinseinkünfte, sondern nach dem Zusammenhang zwischen privater Aneignung und öffentlicher Herausgabe von Steuergeldern. Alternative Zinseinkünfte, die nicht aus Staatskrediten herrühren, sondern aus privater Kreditvergabe, entziehen der Allgemeinheit keine Steuergelder; insofern können sie auch keine umverteilende Wirkung zu ihren Lasten entfalten.

Was »die sozialen Verteilungswirkungen " der kredirfinanzierten Staatsausgaben angeht, die nach Meinung derer zu berücksichtigen sind,"s die die umverteilende Folgewirkung des Inlandskredits in Frage stellen, sollte der Hinweis auf die nicht unbeträchtliche Begünstigung gerade von Selbständigen und Unternehmen aus dem kreditfinanzierten Auftragsschub als Entgegnung genügen.

$\mathrm{Zu}$ berücksichtigen ist überdies, daß die Steuergesetzgebung der letzten 30 Jahre die Beteiligung schwächerer Einkommensschichten an den Zins- und Tilgungsverpflichtungen in Relation zu denen der Unternehmen und der Besserverdienenden verschärft hat: Während die Einnahmen aus der veranlagten Einkommen- und der Körperschaftsteuer 1968 noch $20 \%$ des Gesamts der Steuereinnahmen ausmachten, sind es 1998 nur mehr 6\%. Umgekehrt wuchs der Anteil der Lohnsteuer von 18 auf $32 \%$. Der Umsatzsteueranteil erhöhte sich von 2 auf $25 \% .{ }^{66}$ Lohnsummen-, Vermögen- und Gewerbekapitalsteuer sind inzwischen auf Initiative bzw. unter Mithilfe der Sozialdemokratic von der Bildfläche verschwunden. Infolgedessen sank die Steuerquote zwischen 1980 und 1997 von 25 auf $22 \% .{ }^{57}$ Dies hat nicht unwesentlich zum Anwachsen des Defizits in den öffentlichen Kassen beigetragen; der Zwang zu weiterer Verschuldung stieg.

Das heißt im Ergebnis, daß die Verzinsung der Staatsschuld mittels steigender Belastung der Masseneinkommen finanziert wird, ${ }^{8}{ }^{8}$ während die gleiche Gruppe, die Zinseinkünfte aus Staatskrediten erzielt, ihre Beteiligung am Schuldendienst kontinuierlich mindert.

Zugegeben: Die Motive variieren. War die Staatsverschuldung in Zeiten der sozialliberalen Koalition die sozialdemokratische Antwort auf das Ausbleiben der ver-

52 Ihr Anteil am Schuldenberg belief sich Ende 1997 auf 53 und derjenige an der Nettokreditaufnahme 1997 auf $46 \%$, vgl. Deutsche Bundesbank: Monatsbericht Juni 1998, S. $58^{*}$.

53 Vgl. Schmid 1996, 81 .

54 Gandenberger 1979b, 142. So auch Hofling 1993, 317

ss Puhl r996, 475.

s6 Vgj. Bundesministerium der Finanzen: Finanzbericht 1998, Bonn 1997, Tabelle 12.

57 Jahresgutachten $1997 / 98$ des Sachverstandigenrates zur Begutachtung der gesamtwirtschaftlichen Entwicklung, BT-Drs. 13/9090, Tab. 46

s8 Vgl. auch Arbcitsgruppe Altcrnative Wirtschaftspolitik 1996, 69. 
sprochenen und notwendig gewordenen Umverteilung von oben nach unten, so war sie in der Regierungszeit von Konservativen und Liberalen das folgerichtige Ergebnis der massiven Steuersenkungs- und -streichungspolitik zugunsten von Unternehmen, Einkommens- und Vermögensmillionären. Das verteilungspolitische Ergebnis ist in beiden Motivationslagen das gleiche.

\section{Intergenerative Lastenverschiebung}

"Man sei sich heute einig«, referierte Klaus Lüder von der Verwaltungshochschule Speyer vor einiger Zeit, "daß Verschuldung eine Verschiebung von Lasten von der gegenwärtig lebenden Generation auf künftige Generationen bedeutet. «" Dies trifft im Großen und Ganzen zu. Die ricardianische Sichtweise, wonach Nettokreditaufnahme und Steuern äquivalente Finanzierungsalternativen darstellen und von staatlicher Verschuldung keinerlei intergenerative Umverteilungseffekte ausgehen, ${ }^{60}$ ist wohl endgültig passé. Erhebliche Unterschiede bestehen allerdings hinsichtlich der Bewertung dieser Verteilungswirkung. Während die einen die Ansicht vertreten, mit der Staatsverschuldung würden finanzielle Lasten »unzulässigerweise in die $\mathrm{Zu}$ kunft « verschoben, ${ }^{6 t}$ meinen andere, Staatsverschuldung sorge für sintertemporäre Gerechtigkeit «. ${ }^{62}$

In der sogenannten Normallage dürfen ausschließlich Investitionen aus Krediten finanziert werden. Dahinter steht der Gedanke, daß Investitionen dazu dienen, "die Produktionsmittel der Volkswirtschaft (zu) erhalten, (zu) vermehren oder (zu) verbessern ${ }^{63}$ und daß sie in die Zukunft hineinwirken, $d$. h. von nachfolgenden $\mathrm{Ge}$ nerationen mitbenutzt werden können. Musgrave hat gefolgert, daß die nachwachsenden Generationen überhaupt diejenigen seien, die in erster Linie von staatlichen Investitionen profiticrten. Daher erschien es ihm nur recht und billig, sie an der Finanzierung solcher Ausgaben zu beteiligen; die Kreditfinanzierung diene schließlich dem intertemporalen Lastenausgleich und erweise sich somit als Instrument finanzpolitischer Gerechtigkeit: Zahlungsverpflichtungen verteilten sich - "pay as you use " - über die Jahre der Nutzung der Investition. ${ }^{64}$

Konfrontiert mit der Realität, erweist sich dieses Theorem als reine Apologie einer illegitimen Belastung der Zukunft mit den Folgen der heute vorherrschenden Wachstumsphilosophie. Denn die Ökonomie verhält sich anders, als es das Lehrbuch sich vorstellt. Der Staat tilgt durchaus nicht nutzungsgerecht, sondern er löst mit neuen Krediten die alten ab. Ein Beispiel dafür ist der kreditfinanzierte Dienstwagen, der nach vier Jahren ausgemustert wird und dessen beide Nachfolgemodelle gleichermaßen kreditfinanziert werden. Nach zwölf Jahren zahlen die Steuerpflichrigen Zinsen für drei Kredite, obgleich nur ein Fahrzeug genutzt werden kann. ${ }^{65}$ Es werden also wheute und in Zukunft Zinsen auch für solche Kredite gezahlt (...), deren Gegenwert ganz oder teilweise schon nicht mehr vorhanden ist «. ${ }^{66}$

59 Luder 1994, rf.; im gleichen Sinne auch Puhl 1996, 473 .

$60 \mathrm{Vgl}$. Richter/Wtegard 1993,378

61 Karehnke 1973,405

62 Mußgnug 1996,64

63 Vgl. BT-Drs. V/3040, 47. Schlesinger/Weber/Ziebarth 1993, 212, machen allerdings darauf aufmerksam, daß nur Nettoinvestitionen den offendichen Vermogensbestand mehren.

$64 \mathrm{Vgl}$. Musgrave $1958,72 \mathrm{f}$. Im gleichen Sinne auch Vesper $1996,16$.

65 Vgl. Höfling: mdl. Beitrag auf der Anhörung zur Statsverschuldung, LT(NRW)-Drs. I $2 / 383,8$. Vgl. auch Jahresbericht des Landesrechnungshofs Nordrhein-Westfalen 1990/91, LT(NRW)-Drs. I $1 / 1960,37$.

66 Bundesrechnungshof $1994,23$. 
Auch in vielen anderen Fällen fehlt jede Übereinstimmung zwischen den aus Krediten finanzierten staatlichen Leistungen und dem in der Zukunft aufzubringenden Schuldendienst. Mit Hilfe des öffentlichen Kredits wird folglich überhaupt kein zielgerichteter realer Lastenausgleich zwischen den Generationen hergestellt. ${ }^{67}$ Hinzu kommt, daß der haushaltsrechtliche Investitionsbegriff Erhaltungsinvestitionen mit abdeckt und Folgekosten gänzlich außer Betracht läßt ${ }^{68}$ - was erhebliche (negative) Auswirkungen auf den zukünftigen Kapitalstock in Relation zur Schuldendienstverpflichtung hat.

Was letzten Endes stattfindet, ist eine Reduktion künftiger Realeinkommen ${ }^{69}$ infolge immenser Schuldendienstverpflichtungen und/oder beinahe konfiskatorischer Steuersätze: $7^{\circ}$ Modellrechnungen zufolge haben 1995 Geborene im Durchschnitt netto $21 \%$ ihres Lebensarbeitseinkommens an Steuern zu entrichten, während der Satz für künfrige Generationen bei $55 \%$ liegen dürfte. ${ }^{71}$

Verschiedentlich wird deshalb vorgeschlagen, nur mehr solche Investitionen in die Zukunft zu verlagern, denen sein langfristiges und zukunftsorientiertes Konzept zugrunde liegt «. ${ }^{72}$ Dann wäre immerhin der erwähnte Dienstwagen »erledigt «. ${ }^{73}$ Umweltzcrstörende Projekte wie Autobahnbau, Transrapid, Expansion der Luftverkehrsinfrastruktur usw. würden dadurch allerdings nicht gehindert; im Gegenteil: Die Große Koalition derer, die unbeirrt an die Heilkräfte des "Immer mehr, immer schneller, immer höher « glauben, könnte ihnen ein zusätzliches Unbedenklichkeitszertifikat verpassen: "kreditfinanzierbar, daher zukunftsgerecht".

Die genannten Beispiele weisen auf ein zentrales Problem hin, das die Kreditfinanzierung mit sich bringt: Die Frage nämlich, ob die Vorlieben nachfolgender Generationen die gleichen sein werden wie die der Gegenwart. Erfahrungsgemäß ist dies nicht der Fall. Erinnert sei an den Wasserverkehrswegebau in Frankreich - heute allenfalls Nische für ein eng begrenztes touristisches Segment. Oder: die Bedeutung der Steinkohle. Millionen Menschen wurden für diese "Zukunftsenergie« im Ersten Weltkrieg in den Tod gejagt. Heutc hängen Wallonien, Lothringen, die Saar und das Ruhrrevier am Subventionstropf. Die Deutschen sind froh, niche auch noch die oberschlesischen Bergwerke am Halse zu haben. Und die »Kornkammer Ukraine, für deren zukünftige Ausbeutung abermals Millionen starben? Heute produziert allein Westeuropa Getreide im Übermaß. Die ökologischen Folgekosten unserer aktuellen Produktions-, Distributions- und Konsumtionsweise werden künftige Generationen cbenfalls wenig erfreuen. ${ }^{7+}$ Es ist also schlechterdings unmöglich, gegenwärtige Präferenzen in die Zukunft zu projizieren; wahrscheinlicher ist, daß aus der "kollektiven Erbschaft leicht eine »unliebsame Erblast « wird, die in einer ferneren Zukunft budgetäre Mittel bindet. ${ }^{75}$

Anders stellte sich die Sache dar, wenn die Ausgaben für die Aus- und Fortbildung der Menschen, für die Produktion und Mehrung des mhuman capital «, als Investitionen bewertet würden. Allein die Tatsache, daß die gegcnwärtige Generation der Lernenden identisch ist mit der späteren Generation der Ausgebildeten und sich weiter Bildenden,

$67 \mathrm{Vgl}$. in diesem Sinne Schert 1996,377

68 Tietmeyer 1993, 14.

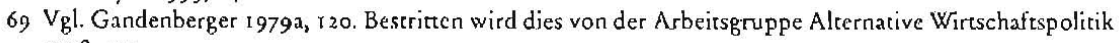
1998,134 .

$70 \mathrm{Vgl}$. Marx 1962,784

7r Vgl. Becker 1998, 4I; Ottnand 1997, 450

72 Becker 1998,35 .

$73 \mathrm{Vgl}$. entsprechende Außenungen der stellvertretenden Vorsitzenden der SI'D-Bundestagsfraktion, Ingrid Maschaus-Milier, in: FAZ vom 20.6.1996.

74 Die jährlichen externen Kosten der Umweltbelastung und-zerstörung werden heute mit $300 \mathrm{Mrd}$. DM angesetzt, vgl. von Weizsacker 1994, 146.

$75 \mathrm{Vgl}$. Wille/Kronenberger $1984,64 \mathrm{I}$. 
macht die Vermutung eines intergenerativen Konsenses plausibel. Das Bundesverfassungsgericht hat zwar entschieden, daß »sich weder aus der Entstehungsgeschichte noch aus Sinn und Z weck «des Art. I s Abs. x Satz 2 Halbs. I GGein Anhal tspunkt für die Bewertung von Bildungsausgaben als Investitionen ergebe; das war aber mehr eine Abwehrhaltung gegen eine befürchtete Ausweitung des Investitionsbegriffs. So ging das Gericht überhaupt nicht cin auf den Zusammenhang zwischen Bildungsausgaben und Zukunftssicherung; es pochte einzig darauf, daß die Einbeziehung von Bildungsaufwendungen der »normativen Intention « der in Redc stehenden Verfassungsbestimmung, "die Staatsverschuldung zu begrenzen, geradewegs zuwiderlaufen " würdc. ${ }^{76}$ Was aber wäre, wenn die Frage, ob Bildungsausgaben als Investitionen anzusehen sind, nicht additiv gestellt würde, sondern alternativ? Hierüber die Diskussion zu eröffnen mit dem Ziel, der Besoldung von LehrerInnen auch verfassungsrechtlich einen höheren Rang einzuräumen als beispielsweise dem Straßcnbau, ist es angesichts der Bildungsmisere in Deutschland bei gleichzeitiger Überfrachtung mit Infrastrukturen für den motorisierten Individualverkehr sicher hohe Zeit.

\section{Reform der Finanzverfassung einleiten}

Ein Schuldenberg, der größer ist als der öffentliche Gesamthaushalt, eine Nettoneuverschuldung, die rechnerisch bloß noch eingegangen wird, um Teile des Zinsendienstes zu begleichen, der wiederum schneller wächst als das Bruttoinlandsprodukt und damit weitere Verschuldung aus sich selbst heraus kreiert: Das ist die Situation der Überschuldung, d.h. der »Veräußerung des Staats. (...) Der einzige Teil des sogenannten Nationalreichtums", der in dieser Lage "wirklich in den Gesamtbesitz der modernen Völker eingeht, ist - die Staatsschuld. «" Deshalb muß jetzt die Grundsatzdebatte über eine definitive Tilgungspolitik eröffnet werden, die gegenwärtig "regelmäßig abgewürgt« wird. $7^{78}$

Zentrale Aufgabe nachhaltiger Finanzpolitik ist es, Handlungsfreiheit wiederzugewinnen - für die unmittelbare und für die fernere Zukunft. Wer die Verkehrs- und Energiewende bewirken, vorsorgenden Umweltschutz etablieren, die moderne Industriegesellschaft umbauen, die sozialen Sicherungssysteme bewahren und ausweiten, Bildung, Weiterbildung und Forschung eincn vorrangigen Platz einräumen will, muß sich von den Fesseln der Staatsverschuldung befreien. Das wird - unter gegebenen Konstellationen - nicht in einem voluntaristischen Akt der Komposition oder der Repudiation möglich sein. ${ }^{79}$ Auch der vereinzelt ins Spiel gebrachte Übergang von verzinslichen Anleihen auf dem Kreditmarkt zu unverzinslichen bei der Bundesbank oder der Europäischen Zcntralbank ${ }^{80}$ empfiehlt sich nicht: Die Gesamtverschuldung mit der Implikation wachsender Tilgungsverpflichtungen in der Zukunft würde weiter steigen.

Ihren Schwung hat die Schuldenakkumulation bekommen, als I 969 die objektbezogenen Regeln für die Kreditaufnahme in Grundgesetz außer Kraft gesetzt wurden. ${ }^{81}$ Die weitere Entwicklung - auch unter Einschluß der Maastrichter Konvergenzkriterien - hat gezcigt, daß dic damals geschaffenen situationsbezogenen Kriterien, jeden-

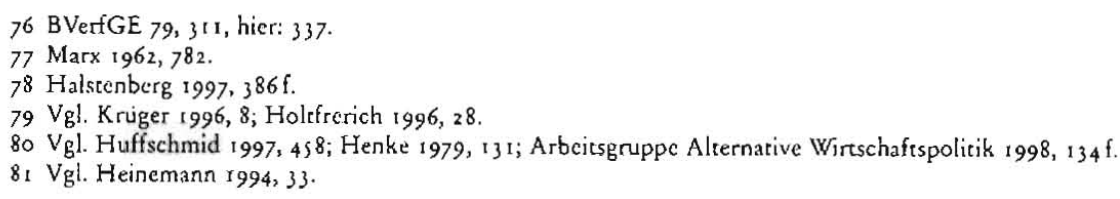


falls in ihrer konkreten Ausprägung in Art. rog Abs. 2 und Irs Abs. I Satz 2 GG, keinen ausreichenden Schutz $z^{8_{2}}$ vor Finanzkrisen bieten. Deshalb dürte es in einem crsten Zugriff erforderlich sein, die heute bestehenden verfassungsrechtlichen Verschuldungsgrenzen enger und klarer zu ziehen, damit die Gesamtverschuldung nicht vollend's aus dem Rudcr läuft. Dies ist bedauerlicherweise im Zuge der einheitsbedingten Verfassungsreform unterblieben, die das Thema Finanzen gänzlich ausgespart hat. ${ }^{\delta_{3}}$

Es geht nicht darum, Kreditaufnahmen verfassungsrechtlich zu verbieten. ${ }^{84}$ Das Recht zur Kreditaufnahme am Kapitalmarkt sollte allerdings auf Fälle konjunkturbedingter Mindereinnahmen und auf unvorhergesehene Sondersituationen (Naturkatastrophen o.̈̈.) beschränkt und mit der Pflicht zur Aufstellung verbindlicher Tilgungspläne verbunden werden. ${ }^{85}$ Wegen der grundsätzlichen Problematik der Verschiebung von Lasten auf die Zukunft sollte das Parlament darlegen müssen, welche Folgekosten zu erwarten sind und inwieweit damit zu rechnen ist, daß die kreditfinanzierten Investitionen auf einen intergenerativen Konsens stoßen. ${ }^{86}$ Präzisiert werden muß, daß ausschließlich Nettoinvestitionen als Bezugsgröße für die Nettoneuverschuldung herangezogen werden dürfen. Dadurch würde sich die Obergrenze für die zulässige Nettokreditaufnahme ohne weiteres halbieren. ${ }^{87}$

Die Ausnahmeregelung des 2. Halbsatzes im zweiten Satz von Art. 1 Is Abs. I GG, wonach zur Abwehr einer Störung des gesamtwirtschaftlichen Gleichgewichts Kredite zur Finanzierung auch konsumtiver Ausgaben aufgenommen werden dürfen, muß fallen: Grenzen bleiben Grenzen. Damit die Neuverschuldung nicht dennoch auf dem Umweg über die Sondervermögen - weiter gedeiht, sind die Haushalte der Sondervermögen etc. bis hin zu privatrechtlich konstruierten Gesellschaften mit öffentlicher Beteiligung in die bisher skizzierten, neu zu schaffenden Kreditobergrenzen einzubeziehen ${ }^{88}$ und der jeweiligen Gebietskörperschaft unmittelbar zuzurechnen. ${ }^{89}$ Um schließlich den Streit zu beenden, inwieweit die Pflicht zur Einhaltung der Kreditobergrenze auch für den Haushaltsvollzug gilt, wird klarzustellen sein, daß die konjunkturbedingte Nettokreditaufnahme die Nettoinvestitionsausgaben weder bei der Aufstellung noch bei der Ausführung des Haushalts überschreiten darf.

"Rein rechtliche Instrumente zur Begrenzung der Staatsverschuldung haben nur begrenzte Reichweite. ${ }^{90} \mathrm{D}$ as mag an der nicht funktionierenden Trennung zwischen Legislative und Exckutive ${ }^{91}$ liegen. Schließlich sind verfassungsrechtliche Hürden nicht schwer zu nehmen, ${ }^{92}$ "weil die Parlamentsmehrheit (...) ihre eigene Regierung

82 Dies meint aber z. B. Karl Georg Zinn: LT(NRW)-Zuschrift $12 / 702,2$

83 Vgl. Fricke 1990,241 .

84 Fur Normalverschuldung Null pladieren von Arnim/Weinberg 1986, 102, 104, und Barbarino 1993, 168. Der Wissenschaftliche Beirat bcim BMF hat in einem Gutachten vom 4.5 .1984 (= Schriftenreihe des Bundesministeriums der Finanzen, Heft 34 , Bonn 1984) ein *Verbot nicht stabilitatspolitisch begründeter regelmäßiger Staatsverschuldung" empfohlen ( 53 ).

85 So auch Stratmann-Mertens: mdl. Beitray auf der Anhönung zur Staatsverschuldung, LT(NRW)-Drs. I $2 /$ 383,48 .

$86 \mathrm{Da}$ es sich dabei bloß um cine Plausibilitatsprüfung handeln kann, ist klar. Dic Darlcgungspflicht durfec dennoch disziplinierende Wirkung entfalten. Daritgungspflichten gegenuber skeptisch: Göke 1996, 7 .

$87 \mathrm{Vgl}$. Höfling: mdl. Beitrag auf der Anhörung zur Staatsverschuldung, LT(NRW)-Drs. $12 / 383$, 3. Außerdem muß in die BHO cin Verbot der heute teilweise noch übliehen, das Gesamtergebnis verfalschenden Doppelzählungen von Investitionsausgaben bei Bund und Landern eingefügt werden, vgl. Brocker 1997, $74 \mathrm{f}$.

88 Art. IIs Abs. 2 GG ware zu streichen.

89 Vgl. dazu Publ 1996, 512 f.

90 Stratmann-Mertens: Schrifeliche Stellungnahme zum Fragenkatalog für die A nhörung des Haushalts- und Finanzausschusses des Landtags Nordrhein-Westfalen zur Staatsverschuidung am 31.10.1996, LT(NRW)-Zuschrift $12 / 765,2$.

91 Vgl. Gantner 1979, $147 \mathrm{f}$.

$92 \mathrm{Vgl}$. Goke 1996, 4 
in der Regel nicht « kontrolliert, sondern nach außen abschirmt. ${ }^{93}$ Untersuchungen in den USA haben jedenfalls zutage gefördert, daß selbst Bundesstaaten mit rigorosen Kreditlimits hohe Schuldenstände aufweisen..$^{94} \mathrm{Um}$ dieser Gefahr vorzubeugen, wird es erforderlich sein, ein Gremium/eine Institution zu schaffen, das/die ein Gegengewicht gegen Fotderungen bildet, »die immer von den verschiedenen Seiten erhoben werden $\propto .95$ Als eine solche Instanz außcrhalb von Regierung und Parlament ${ }^{96}$ kämen will man/frau nicht eine neue Einrichtung schaffen - die Rechnungshöfe oder die Bundesschuldenverwaltung in Betracht. Wichtig wäre, das entscheidende Personal in gleicher Weise wic RichterInnen am Bundesverfassungsgericht bzw. an den Verfassungsgerichtshöfen der Länder auszuwählen und einzusetzen, es mit richterlicher Unabhängigkeit auszustatten (ist bei den Rechnungshöfen bereits gegeben) und auf Dauer zu bestallen. Dieses Gremium - bzw. diese Gremien, falls vorgesehen werden sollte, sie gesondert auch auf Länderebene einzusetzen - hätte, unter Berücksichtigung der verfassungsrechtlichen Vorgaben, unmittelbar und endgültig positiv oder negativ über eine geplante Nettokreditermächtigung zu entscheiden.

Nur eine solche Kombination aus Verfassungsreform und Kontrollgremium dürfte geeignet sein, dem weiteren Marsch in die »Veräußerung des Staates« und seiner Auslieferung an international operierende Finanzkomplexe Einhalt zu gebieten. Im Laufe der Zeit ${ }^{97}$ könnten die Vorschläge dazu beitragen, an den Schuldendienst verlorene Handlungsspielräume zurückzugewinnen, Reformpolitik wieder finanzierbar zu machen und die fiskalische Belastung späterer Generationen mit der Wirtschaftsweise unserer maßlosen Gegenwart zu mindern.

\section{Und woher kommt das Geld?}

Dem Verschuldungsabbau, den sie in Lehrbüchern und Sachverständigengutachten fordert, den ihre politischen Vollstrecker indes nicht betrieben, stellt die neoliberale Ideologie die Demontage des staatlichen Aufgaben- und Leistungskatalogs an die Seite. Es müsse allseitig gespart, der Staat auf seine "eigentlichen Aufgaben " (den Repressionsapparat) zurückgeführt und die öffentliche Verwaltung auf einen »schlanken « Rest geschrumpft werden. Auf diesem Hintergrund ist es nicht verwunderlich, daß manche, hören sie vom "Abbau der Staatsverschuldung “ reden, "Abbau des Sozialstaats « verstehen. ${ }^{98}$ Tatsächlich geht es indes darum, der ansonsten unweigerlich auf die Gesellschaft zukommenden Situation vorzubeugen, daß die Realeinkommen breiter Einkommensschichten allein wegen der aufgehäuften Schulden und den daraus erwachsenden Zinslasten dramatisch sinken.

Richtig an der neoliberalen Forderung nach Einsparungen ist, daß staatliche Ausgaben und Subventionen auf den Prüfstand gehören: Abschreibungserleichterungen im Steuerrecht, das Fehlen einer Quellenbesteuerung von Kapitaleinkommen und der weitgehende Verzicht auf die Besteuerung von Gewinnen aus Kauf und Verkauf von

93 Schuster 1971, 137

94 von Hagen i $99 \mathrm{~s}$

95 Ex-Bundesbankprásident Karl Blessing, zit. n. Nowotny 1979, 10. Blessing hatte das Zicierte allerdings mit Blick auf die Unabhängigkeit der Bundesbank geaußert.

$96 \mathrm{Vgl}$. Blasius/Jahne 1997, 34.

97 Wic weit der Weg ist, hat Becker 1998, r24, an cinem Beispiclsfall vorgercchnet. Danach dauerte es bei einer jahrlichen Tilgung von ro Mrd. DM und \%usätzlicher Zinsendienstleistung tro Jahre, bis der gegenwärtige Schuldenberg des öffentlichen Gesamthaushalts halbier wärc.

98 Manche wittern in diesem Zusammenhang xoko-calvinistischen Konsumverzicht«. Vgl. Herzberg/Hoff mann/Mostofizadeh 1998,29 
Immobilien, Wcrtpapieren, Devisen und Optionen, die Steinkohlebeihilfen, die Unterstützung von Konzernen bei der Hochtcchnologieforschung und -anwendung, die Förderung tierquälerischer, grundwasserbelastender und klimaschädigender Massenviehhaltung und so weiter und so fort. Wichtig ist auch, den wachsenden Versorgungslasten bei Bund, Ländern und Gemeinden entgegenzuarbeiten. Dabei sind jedenfalls für die kleinen und mittleren Einkommen - weitere Leistungskürzungen und Verlängerungen von Anwartschaftszeiten nicht mehr vertret- und wahrscheinlich kaum mehr durchsetzbar. Die ständische Klassifizierung in ArbeiterInnen, Angestellte und BeamtInnen ist längst überholt und sollte einem einheitlichen öffentlichen Personalrccht weichen, bei dem die Altcrsversorgung aus aktuell zu leistenden Beitragszahlungen und nicht aus gegebenen Versprechen finanziert wird.

Dennoch: Sofern der historisch gewachsene, in jahrzehntelangen Auseinandersetzungen und Übereinkünften austarierte Aufgaben- und Leistungskatalog des Sozialstaats Bundesrepublik, der verfassungsrechtlich überdies zum "Schutz der natürlichen Lebensgrundlagen « verpflichtet ist (Art. 20a GG), nicht gegenreformerisch ausgehöhlt werden soll, stoßen Einsparungen bald an Grenzen. Die, auch in bezug auf ihr Volumen, einzig realistische Alternative zur Staatsverschuldung ist die Schließung des nach Einsparungen verbleibenden staatlichen Finanzierungsdefizits durch Steuern. ${ }^{99}$ Wer hiergegen einwendet, die »Flucht in Steuererhöhungen« sei »kein geeigneter Weg ${ }^{100}$ weil dadurch negativere Wirkungen einträten, als wenn die Verschuldung in einem bestimmten Rahmen gehalten würde, ${ }^{101}$ vergißs, daß z.B. Karl Schillers Konzept einer aus Steuern finanzierten Erhöhung der Staatsausgaben einen expansiven Effekt erzielte, der aus der Rezession $1966 / 67$ herausführte. ${ }^{102}$ Hcute ist zudem weitestgehend unumstritten, daß die deutsche Einheit besser aus Steuererhöhungen als im Wege exorbitanter Neuverschuldung finanziert worden wäre. ${ }^{103}$ Auch in der gegenswärtigen Situation ist eine Aufkommensmehrung unverzichtbar. Denn es kann überhaupt nicht ernsthaft bezweifelt werden, daß der Staat mehr Geld braucht, wenn er nicht seinen Aufgaben- und Leistungskatalog - auch zu Lasten feministischer, bürgerInnenrechtlicher, ökologischer Projekte - eindampfen soll.

Allerdings können kleine und mittlere Einkommen, vor allem, wenn sie zusätzlich mit Beiträgen zu den gesetzlichen Sozialversicherungen belastet sind, keine weiteren Abgabenerhöhungen verkraften. Da das Gemeinwesen aber zusätzlicher Mittel bedarf, wird kein Weg daran vorbeiführen, diese in erster Linie dort abzurufen, wo bisher eine, gemessen an deren Leistungskraft, unterdurchschnittliche Steuerbelastung vorliegt: bei den BezicherInnen großer Einkommen, den Vermögenden und den großen Unternehmen. ${ }^{104}$ Gerade ihnen fällt es aber in einer Welt deregulierter Kapi-

$99 \mathrm{Vgl.} \mathrm{auch} \mathrm{Rügemer} 1995,57$.

100 Singer 1996b, 32

I01 Vgl. Klaus Gretschmann: mdl. Beitrag auf der Anhơning zur Staatsverschuldung, LT(NRW)-Drs. 12/ $383,19$.

102 Vgl. Karl Georg Zinn: mdi. Beitrag auf der Anhonng zur Staatsverschuldung, LT(NRW)-Drs. 1 $2 / 38_{3}$, 24 .

$103 \mathrm{Vgl}$. Offentliche Haushalte 1997/98. Trotk restriktiver Ausgabenpolitik weiterhin hohe Defizite, in: DIW-Wochenbericht 64. 1997, 647-662, 647. Selbst die Bundesbank kritisiert, daß sich die KohlRegierung mit ihren einnahmepolitischen "Entscheidungen nur unzureichend auf die neue Situation cin(gestellt hätte), was zur Folge hatte, daß letztlich zu einem betrachelichen Teil auf die Kreditfinanzicrung ausgewichen werden mußte* (Monatsberieht Marz 1997, 19).

${ }_{10}$ Die Umverteilung der Einkommen durch die Finanzpolitik zugunsren der Unternehmen und der Gewinumeinkommensbezieher bestatige DIW-Wochenbericht 50. 1993, 507. Die Landesregierung Baden-Wurttembers hat erreehnet, daß für Einkünfte zwischen 0,2 s und I Mio. DM in der Praxis bloß I 4 bis $28 \%$ Steuern fallig werden; vgl. Die Woche vom 24.4. I 998 . Einer Untersuchung des Mannheimer Zentrums für Europäische Wirtsehaftsforschung zufolge zahlen Haushalte, die sich an der Spitze der Einkommenspyramide befinden, nur mehr einen auf das Bruttoeinkommen bezogenen Grenzsrcuersatz von $32 \%$; vgl. Huster 1996,21 . 
talmärkte und der Transnationalisierung der Ökonomie nicht schwer, sich nationalen Steuergesetzen sogar legal zu entziehen. Dies stumpft das Schwert der Einkommenbesteuerung erheblich ab - vor allem, da realistischerweise keine Rückkehr zum status quo ante zu erwarten ist. ${ }^{\text {10 }}$

Deshalb werden die in der Bundesrepublik spiegelbildlich zum öffentlichen Schuldenberg angehäuften Vermögen als Bemessungsgrundlage einer Tilgungssteuer herangezogen werden müssen. ${ }^{106}$ Allein die privaten Haushalte in der Bundesrepublik verfügen über ein Gesamtvermögen von ${ }_{13}$ Billionen DM, dessen größter Teil sich im Eigentum weniger befindet. Dazu kommen betriebliche Vermögen in etwa der gleichen Höhe. ${ }^{107}$ Von diesen Vermögen müßte - in Anlehnung an entsprechende Vorbilder bei der Konzeption des Lastenausgleichsgesetzes von r952 - für einen befristeten Zeitraum ein anschnlicher Betrag mobilisiert werden. Er müßte mindestens so hoch sein, daß er die Zinsausgaben deckt und zusätzlich Mittel zur Tilgung des aufgetürmten Schuldenberges übrigbleiben. Einwände hiergegen sollten nicht unberücksichtigt lassen, daß dieser Vorschlag ein gutes Stück Gerechtigkeit für sich hat: Weil eine beträchtliche personelle bzw. institutionelle Übereinstimmung zwischen GläubigerInnen und Tilgungssteuerpflichtigen bestehen dürfte, flössen die Steuereinnahmen in Form von Tilgungsbeiträgen weitestgehend an die SteuerschuldnerInnen zurück. Es ergäbe sich dadurch aber, gleichsam ohne Entzug von Kaufkraft oder Investitionsfähigkeit, eine schrittweise Entschuldung des öffentlichen Gesamthaushalts, die ihn in einen zukünfrig tragfähigen Zustand versetzen könnte. Eine andere, vertretbare Alternative zur Bewältigung des Problems der Staatsverschuldung ist nicht in Sicht.

\section{Literatur}

Andel, Norbert, Zur These von den unsozialen Verteilungswirkungen der öffentlichen Schulden, in: Public Financc 24. 1969, 69-77.

Arbeitsgruppe Alternative Wirtschaftspolitik, Gegen kapitalorientierte Standortdebatte und Zeitdiktat bei der Europäischen Währungsunion. Memorandum '96, Köln 1996.

dies., Bewegung in Europa, Blockade in Deutschland - Kurswechsel für Beschäfrigung. Memorandum ' 98 , Köln 1998 .

Arnim, Hans-Herbert von, Dagmar Weinberg, Staatsverschuldung in der Bundesrepublik Deutschland, Wiesbaden 1986.

Barbarino, Otto, Die unzureichende Wende, Frankfurt a. M. 1993.

Becker, Andreas, Staatsverschuldung und Umweltzerstörung - wie sie vereint die Zukunft berauben, München 1998 .

Bischoff, Joachim, Politik in einer reichen Gesellschaft, in: Hcrbert Schui, Eckart Spoo (Hrsg.): Geld ist genug da. Reichtum in Deurschland, Heilbronn 1996, $230-235$.

Blasius, Hans, Eduard Hans Jahnz, Staatsschulden (Teil 3), in: Verwaltung und Management 3. I997, 31-37.

Boss, Alfred, Jens Oliver Lorz, Die öffentliche Verschuldung in der Bundesrepublik Deutschland. Ausmaß und Belastungswirkung, in: Jahrbuch für Wirtschaftswissenschaften ${ }_{4} 6$. I995, I5 2-183.

Brenner, Georg, Carl E. Haury, Ernst-Moritz Lipp, Staatsverschuldung und Verfassung, in: Finanzarchiv 38 . $\times 980,236-255$.

ios Vgl. Graf 1996, s7

$106 \mathrm{Vgl}$. Lorenz Jarass: mdl. Beitrag auf der Anhörung zur Staatsverschuldung, LT(NRW)-Drs. $12 / 383,33$. Ganz entschieden dagegen, weil damit die Volkswirtschaft zusatzlich belaster wurdc, Boss/Lorz 1995 , 179 .

10) Vgl. Die Woche vom 24.4.1998; Bundnis 9o/Die Grunen, Bundestagsfrakrion: Okologisch gestaleen, soziale Gerechtigkeit wahren und kommende Generationen entlasten - die bundnisgrunen Antworten auf das Sparpaket der Bundesregierung. Argumente und Fakten, Bonn 1996, 12; 10\% der privaten Haushalte verfugen über $49 \%$ des Vermogens: vgl. Bischoff 1996,231 . 
Bröcker, Klaus T., Grenzen staatlicher Verschuldung im System des Verfassungsstaats, Berlin 1997.

Bundesrechnungshof: Bemerkungen 1994 zur Haushalts- und Wirtschaftsführung (einschließlich der Feststellungen zur Jahresrechnung des Bundes 1992), BT-Drucksache 12/ 8490 .

Domar, Evsey D., The »Burden of the Debt " and the National Income, in: American Economic Review 34. 1944, 798-827.

Edinger, Florian, Private Vorfinanzierung öffentlicher Investitionen und Haushaltsrecht, in: Zcitschrift für Gesetzgebung 12. 1997, 242-256.

Fischer-Menshausen, Herbert, Arrikel irs GG, in: Ingo von Münch, Philip Kunig (Hrsg.): Grundgesetz-Kommentar, 3. Aufl. München 1996, r209-1238.

Friauf, Karl Heinrich, Staatskredit, in: Josef Isensee, Paul Kirchhof (Hrsg.): Handbuch des Staatsrechts der Bundesrepublik Deutschland, Bd. IV, Heidelberg 1990, 321-355.

Fricke, Eberhard, Kreditbegrenzung im Staatshaushalt - Eine vertane Chance dcs Bundesgesetzgebers, in: Finanzarchiv n.F. $4^{8}$. 1990, 222-243.

Gandenberger, Otto, Der öffentliche Kredit in außerkonjunktureller Betrachtung, in: Evald Noworny (Hrsg.): Öffentliche Verschuldung, Stuttgart/New York 1979a, $117-124$.

ders., Dic Wirkung des öffentlichen Kredits auf die Einkommensverteilung, in: Ewald Nowotny (Hrsg.): Öffendiche Verschuldung, Stuttgart/New York 1979b, I $38-144$.

ders., Theorie der öffentlichen Verschuldung, in: Fritz Neumark (Hrsg.): Handbuch der Finanzwissenschaft, Bd. III, 3. Aufl. Tübingen I98 x, 3-49.

Gantner, Manfried, Zur Frage nach den Grenzen der öffentlichen Verschuldung, in: Ewald Nowotny (Hrsg.): Öffentliche Verschuldung, Stuttgart/New York 1979, S. 145-161.

Göke, Wolfgang, Staatsverschuldung. Finanzverfassungsrechtliche Ursachen und Lösungen zu ihrer Begrenzung, in: NdsVBI. 3. 1996, 1-8.

Graf, Ralph, Das Elend der Verschuldung, in: Kommune 14. 1996, Nr. 3, 56-57.

Hagen, Jürgen von, A Note on the Empirical Effectiveness of Formal Fiscal Restraints, in: Journal of Public Economics 44. 1991 , 199-210.

Halstenberg, Friedrich, Finanzplanung und Staatsverschuldung, in: Franz Joscf Düwell (Hrsg.): Anwalt des Rechtsstaats. Festschrift für Diether Posser zum 75. Geburtstag, Köln 1997, $385-$ 394.

Hardt, Ulrike, Uwe Hiersemenzel, Horst Keppler, Verschuldungsgrenzen für die öffentlichen Haushaltc. Einige ökonomische Überlegungen anläßlich der niedersächsischen Verfassungsdebatte, in: NdsVBI. 3. 1996, 25-33.

Hartmann, Uwe, Europäische Union und die Budgetautonomie der deutschen Länder, BadenBaden 1994.

Hauser, Hans-Joachim, Verteilungswirkungen der Staatsverschuldung. Eine kreislauftheoretische Inzidenzbetrachung, Frankfurt a. M./Bern/Las Vegas 1979.

Heinemann, Friedrich, Staatsverschuldung. Ursachen und Begrenzung, Köln 1994

Henke, Klaus-Dirk, Kreditaufnahme und Besteuerung als Alternativen der Infrastrukturfinanzierung, in: Ewald Nowotny (Hrsg.): Öffentliche Verschuldung, Stuttgart/New York 1979, $125-137$.

Henneke, Hans-Günter, Not kennt kein Gebot und macht erfinderisch: Gesetzgeberische Gestaltungsspielräume und Darlegungslasten bei der Nettokreditaufnahme, in: NdsVBl. 4. 1997, 217-225.

Herzberg, Markus, Barbara Hoffmann, Mehrdad Mostofizadeh, NRW-Linke: Genuß und Kritik, in: Andere Zeiten, Nir. 2 1998, 26-29.

Heuer, Kommentar zum Bundeshaushaltsrecht, Bd. I, Neuwied (Stand: Mai 1998).

Heun, Werner, Staatsverschuldung und Grundgesetz, in: Die Verwaltung x. 1985, 1-28.

Höfling, Wolfram, Staatsschuldenrecht. Rechtsgrundlagen und Rechtsmaßstäbe für die Staatsschuldenpolitik in der Bundesrepublik Deutschland, Heidelberg 1993.

Holtfrerich, Carl-Ludwig, Bewältigung der deutschen Staatsbankrotte 1918 und 1945, in: Erhard Kantzenbach (Hrsg.): Staatsüberschuldung, Göttingen 1996, 27-57.

Huffschmid, Jörg, Ursachen, Funktion und Nutznießer der Staatsverschuldung, in: Blättcr für deutsche und internationalc Politik 42. 1997, 454-459.

Karehnke, Helmut, Zur Änderung des Artikels i is des Grundgesetzes, in: DÖV 26. $1973,393-$ 406.

Kirchhof, Paul, Grenzen der Staatsverschuldung in einem demokratischen Rechtsstaat, in: Hans-Herbert von Arnim, Konrad Littmann (Hrsg.): Finanzpolitik im Umbruch. Zur Konsolidierung öffentlicher Haushalte, Berlin $1984,271-28 \mathrm{r}$.

ders., Staatliche Einnahmen, in: Josef Isensce, Paul Kirchhof (Hrsg.): Handbuch des Staatsrechrs der Bundesrepublik Deutschland, Bd. IV, Heidelberg 1990, 87-233. 
Kitterer, Wolfgang, Über die Tragbarkeit und die Grenzen der Staatsverschuldung, in: Erhard Kantzenbach (Hrsg.): Staatsüberschuldung, Göttingen 1996, 79-89.

Krüger, Malte, Staatsbankrotte: Es gab sie, und es wird sie geben, in: Orientierungen zur Wirtschafts- und Gesellschaftspolitik 68. 1996, 8-13.

Lerner, Abba P., Everybody's Business, New York 1964

Lüder, Klaus, Ist das Land Nordrhein-Westfalen finanziel! ruiniert? Vortrag anlässlich der Tagung "Innovation für NRW - Politik und Verwaltung im Diskurs* der Fortbildungsakademie des Innenministeriums des Landes Nordrhein-Westfalen am r. x 2. 1994 in Oer-Erkenschwick (unveröff. Ms.).

Marx, Karl, Das Kapital, Bd. I (=Marx-Engels-Werke Bd. 23), Berlin I 962.

Mattick, Paul, Marx und Keynes. Die Grenzen des "gemischten Wirtschaftssystems", Frankfurt a. M./Köln 1971 .

Maunz, Theodor, Art. ıs GG, in: ders., Günter Dürig: Grundgesetz. Loseblattkommentar (Stand: 33. Lfg. 1997).

Mehde, Veith, Gesetzgebungskompetenz des Bundes zur Aufteilung der Verschuldungsgrenzen des Vertrages von Maastricht?, in: DÖV so. 1997, 616-624.

Müller, Udo, Die Geltung der verfassungsrechtlichen Kredithöchstgrenze des Art. I Is Abs. I Satz 2 GG im Yaushaltsvollzug, in: DÖV 49. 1996, 490-497.

Musgrave, Richard A., Theorie der öffentlichen Schuld, in: Wilhelm Gerloff, Fritz Neumark (Hrsg.): Handbuch der Finanzwissenschaft, Bd. III, Tübingen 1958, 68-137.

Mußgnug, Reinhard, Staatsüberschuldung und Verfassungsrecht, in: Erhard Kantzenbach (Hrsg.): Staatsüberschuldung, Göttingen 1996, 59-78.

Nahamowitz, Peter, Die verfassungsrechtlichen Grenzen der Staatsverschuldung, in: DuR I 8. I $990,427-443$.

Nowotny, Ewald, Zur politischen Ökonomie der öffentliehen Verschuldung - eine Einführung, in: ders. (Hrsg.): Öffentliche Verschuldung, Stuttgart/New York 1979, 1-16.

Oberhauser, Alois, Das Schuldenparadox, in: Jahrbücher für Nationalökonomie und Statistik 200. $1985,333-348$.

Ottnand, Adrian, Die Staatsverschuldung wächst nach eigenen Gesetzen, in: Orientierungen zur Wirtschafts- und Gesellschaftspolitik 68. 1996, 2-7.

ders., Wohlstand auf Pump. Der Irrweg der Schuldenpolitik, in: Blätter für deutsche und internationale Politik 42. 1997, 444-453.

Patzig, Werner, Das Haushaltsrecht des Bundes und der Länder. Kommentar zu den Rechts- und Verwaltungsvorschriften, Bd. II, Baden-Baden 1982 (Stand: 8. Lfg. I99r).

Piduch, Erwin Adolf, Bundeshaushaltsrecht, 2. Aufl. Stuttgart (Stand: 34. Lfg. 1997).

Pohmer, Dieter, Die Staatsverschuldung als Instrument der Verteilungspolitik, in: Heinz Haller, Willi Albers (Hrsg.): Probleme der Staatsverschuldung, Berlin 1972, 143-187.

Prokisch, Rainer, Die Justiziabilität der Finanzverfassung, Baden-Baden 1993.

Puhl, Thomas, Budgetflucht und Haushaltsverfassung, Tübingen 1996.

Richter, Wolfram F., Wolfgang Wiegard, Zwanzig Jahre "Neue Finanzwissenschaft«, Teil II: Steuern und Staatsverschuldung, in: Zeitschrift für Wirtschafts- und Sozialwissenschaften Ir 3. 1993, 337-400.

Rügemer, Werner, Der Staat ist cine begehrte Schuldneradresse, in: Kommune r 3. 1995, Nr. I r, $53-57$.

Schemmel, Lothar, Defizitbegrenzung im Bundesstaat. Umsetzung des europäischen Stabilitätspakts bei Bund und Ländern, Wiesbaden 1997.

Scherf, Wolfgang, Politische Ursachen und Möglichkeiten einer konstitutionellen Begrenzung der staatlichen Neuverschuldung, in: Staatswissenschaften und Staatspraxis 7. 1996, 365386 .

Schieren, Stefan, Armut im Reichtum. Besteuerungswirklichkeit in Deutschland, in: Soziaic Sicherheit 43. 1994, 201-2rI.

Schlesinger, Helmut, Manfred Weber, Gerhard Ziebarth, Staatsverschuldung - ohne Ende? Zur Rationalität und Problematik des öffentlichen Kredits, Darmstadt 1993.

Schmahl, Hans-Jürgen, Struktur des Staatsdefizits und Konsolidierungspolitik, in: Erhard Kantzenbach (Hrsg.): Staatsüberschuldung, Göttingen 1996, 91-I02.

Schmid, Fred, Finanzspekulation, in: Herbert Schui, Eckart Spoo (Hrsg.): Geld ist genug da. Reichtum in Deutschland, Heilbronn 1996, 78-8s.

Schuster, Rudolf, Ein neues Grundgesetz? Überlegungen zur geforderten Verfassungsreform, in: Gerhard Lehmbruch, Klaus von Beyme, Iring Fetscher (Hrsg.): Demokratisches System und politische Praxis in der Bundesrepublik, München 1971, 127-142.

Singer, Otto, Schulden, die nicht vergehen wollen, in: Kommune 14. 1996a, Nr. 1, 33-36.

ders., Weiter in der Schuldenfalle, in: Kommune 14. 1996b, Nr. 5, S. 3 I-34. 
Sturm, Roland, Staarsverschuldung. Ursachen, Wirkungen und Grenzen staatlicher Verschuldungspolitik, Opladen 1993.

Tiemann, Susanne, Die verfassungsrechrliche Kreditobergrenze im Haushaltsvollzug, in: DÖV 48. $1995,632-636$.

Tietmeyer, Hans, In welchem Umfang darf sich ein Staat verschulden?, in: Aus Politik und Zeitgeschichte 1993, B I8, 13-18.

Vesper, Dieter, Staatsbankrott - gibt's den?, in: Ästherik und Kommunikation 1996, Nr. 93, 13I 8.

Walters, Alan Arthur, How to Make a Benefit of the Burden of National Debt, in: National Tax Journal 20. 1967, 316-318.

Watrin, Christian, Keynes' Sozialphilosophie und ihre wirtschaftspolitischen Implikationen, in: Wirtschaftsdienst 76. 1996, 229-232.

Weizsäcker, Ernst Ulrich von, Erdpolitik. Ökologische Realpolitik an der Schwelle zum Jahrhundert der Umwelt, 4. Aufl. Darmstadt 1994.

Wiese, Jörg A., Ist die Staatsverschuldung in Europa tragbar? in: Orientierungen zur Wirtschafts- und Gesellschaftspolitik 71 . 1997, 52-58.

Wille, Eberhard, Stefan Kronenberger, Zielkonflikte im Kontext der Staatsverschuldung. Einige Anmerkungen mit empirischem Bezug, in: Horst Siebert (Hrsg.): Intertemporale Allokation, Frankfurt a.M. $1984,607-647$. 


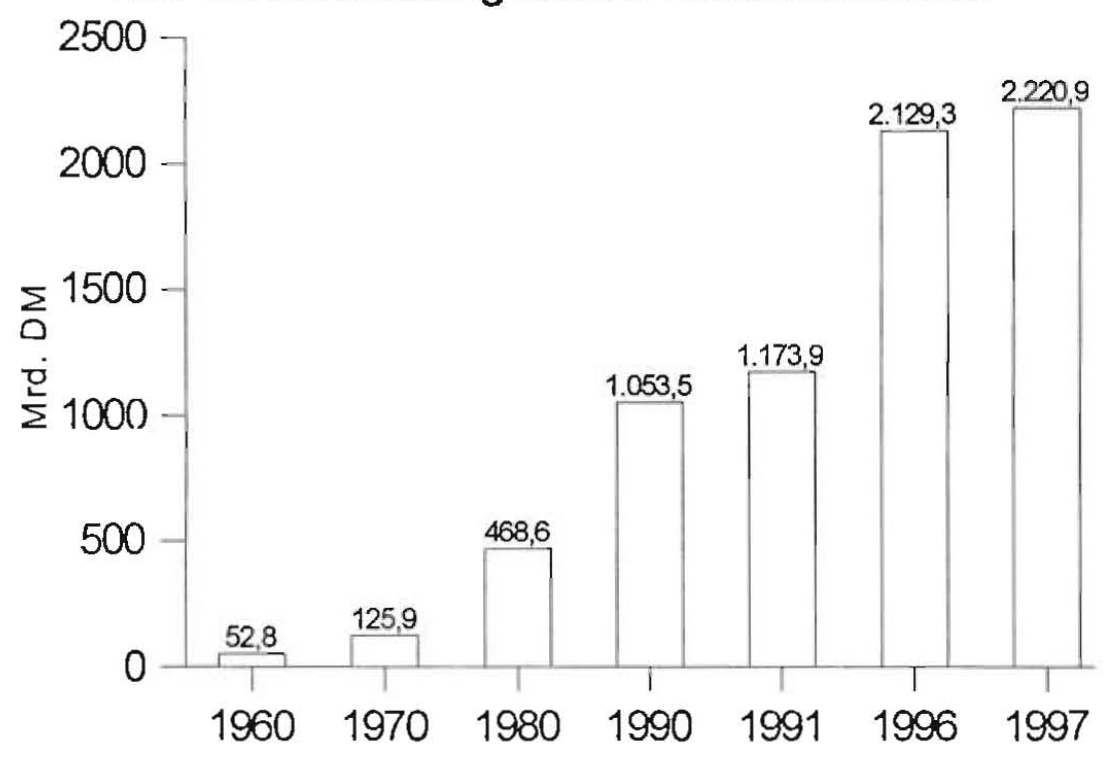

Quelle: Deutsche Bundesbank

Tab. 2: Nettokreditaufnahme und Zinsausgaben des Bundes und der Länder

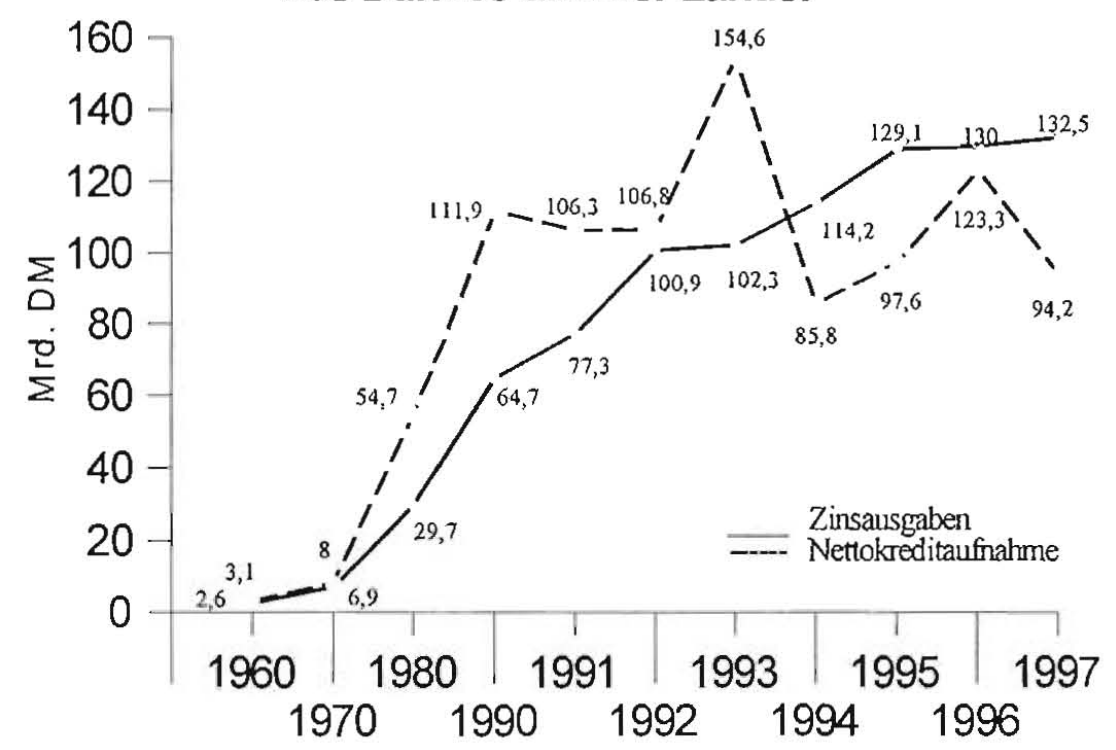

Quetle: Deutsche Bundesbank 
Tab. 3: Zins-Steuer-Quoten

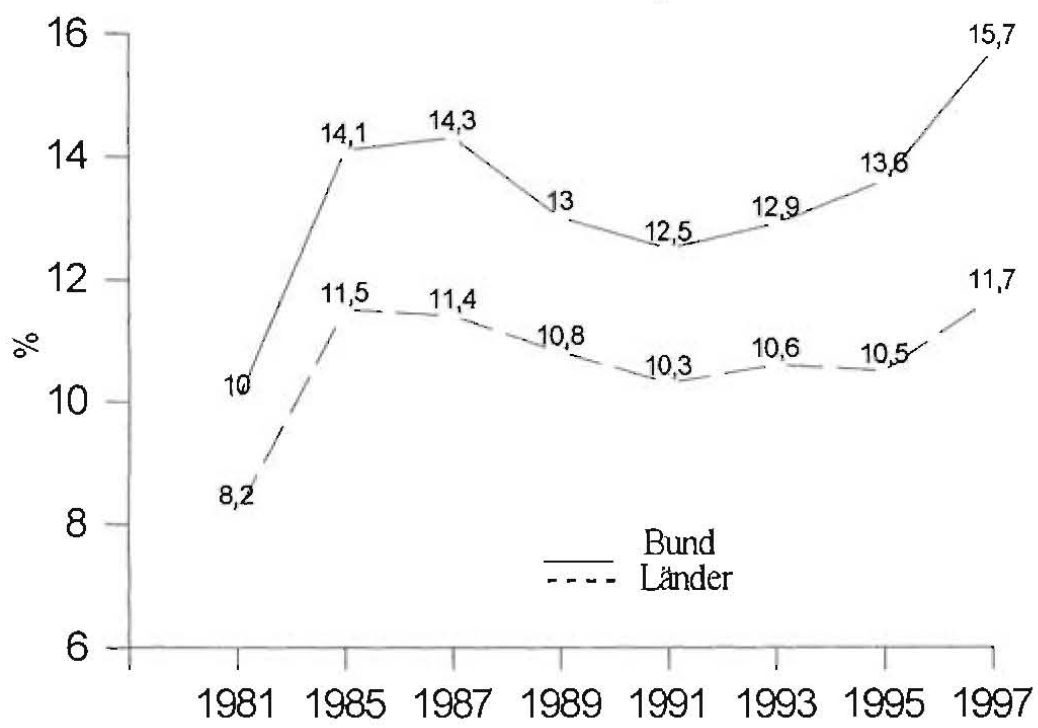

Quelle: Finanzbcricle NRW 1997, 1998; LT (NRW) Drs. 12/1201 urid 12/2401

Tab. 4: Auslandsverschuldung

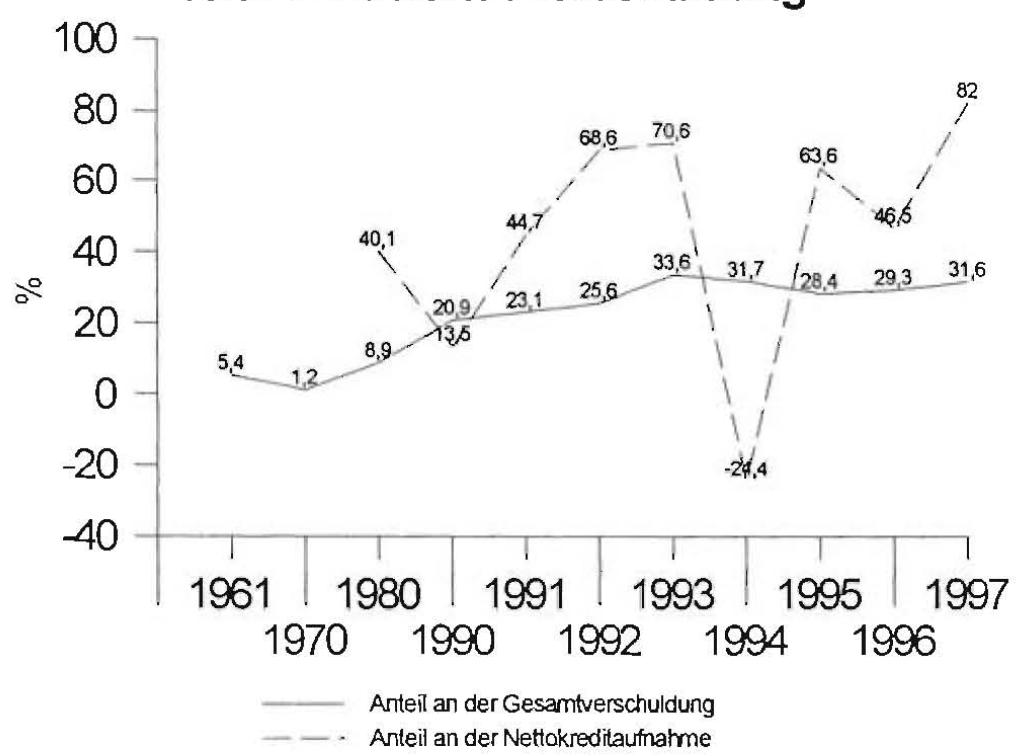

Quelle: Deutsche Bundesbank 